\title{
Identification of a 13-gene-based classifier as a potential biomarker to predict the effects of fluorouracil-based chemotherapy in colorectal cancer
}

\author{
ZUHUAN GAN $^{1 *}$, QIYUAN ZOU ${ }^{2 *}$, YAN LIN $^{3}$, ZIHAI XU $^{1}$, \\ ZHONG HUANG ${ }^{1}$, ZHICHAO CHEN ${ }^{1}$ and YUFENG LV ${ }^{1}$

\begin{abstract}
Departments of ${ }^{1}$ Medical Oncology and ${ }^{2}$ Medicine, Affiliated Langdong Hospital of Guangxi Medical University, Guangxi Medical University Kaiyuan Langdong Hospital; ${ }^{3}$ Department of Medical Oncology,

Affiliated Tumor Hospital of Guangxi Medical University, Nanning,

Guangxi Zhuang Autonomous Region 530021, P.R. China
\end{abstract}

Received October 19, 2018; Accepted February 25, 2019

DOI: $10.3892 / \mathrm{ol} .2019 .10159$

\begin{abstract}
The aim of the current study was to develop a predictor classifier for response to fluorouracil-based chemotherapy in patients with advanced colorectal cancer (CRC) using microarray gene expression profiles of primary CRC tissues. Using two expression profiles downloaded from the Gene Expression Omnibus database, differentially expressed genes (DEGs) between responders and non-responders to fluorouracil-based chemotherapy were identified. A total of 791 DEGs, including 303 that were upregulated and 488 that were downregulated in responders, were identified. Functional enrichment analysis revealed that the DEGs were primarily involved in 'cell mitosis', 'DNA replication' and 'cell cycle' signaling pathways. Following feature selection using two methods, a random forest classifier for response to fluorouracil-based chemotherapy with 13 DEGs was constructed. The accuracy of the 13-gene classifier was 0.930 in the training set and 0.810 in the validation set. The receiver operating characteristic curve analysis revealed that the area under the curve was 1.000 in the training set and 0.873 in the validation set $(\mathrm{P}=0.227)$. The 13-gene-based classifier described in the current study may be used as a potential biomarker to predict the effects of fluorouracil-based chemotherapy in patients with CRC.
\end{abstract}

Correspondence to: Dr Yufeng Lv, Department of Medical Oncology, Affiliated Langdong Hospital of Guangxi Medical University, Guangxi Medical University Kaiyuan Langdong Hospital, 60 Xiangbin Road, Nanning, Guangxi Zhuang Autonomous Region 530021, P.R. China

E-mail: tougaozhuanyong_lv@126.com

${ }^{*}$ Contributed equally

Key words: colorectal cancer, fluorouracil-based chemotherapy, differential expression genes, random forest classifier

\section{Introduction}

Colorectal cancer (CRC) is the third most commonly diagnosed cancer in males and the second in females, and it is one of the most common causes of cancer mortality (1). Localized CRCs are amenable to curative surgical resection, however, $\sim 25 \%$ of patients present with metastatic disease and $\sim 50 \%$ of patients will develop metastases (2). Fluorouracil-based chemotherapy remains the primary treatment for metastatic CRC (3). 5-fluorouracil (5-FU) alone has an objective response rate of $20 \%$ (4). The addition of irinotecan or oxaliplatin to 5-FU increases the objective response rate to $\sim 50 \%$ (5). The effects of 5-FU/leucovorin combined with irinotecan (FOLFIRI) or oxaliplatin (FOLFOX) in the first-line treatment of metastatic CRC are comparable (6). In the last decade, the addition of targeted therapies based on these chemotherapy regimens has improved the therapeutic approach and significantly increased progression-free survival and overall survival times (7-9). Fluorouracil-based chemotherapy remains the primary treatment for metastatic CRC. However, $\sim 50 \%$ of patients are resistant to fluorouracil-based chemotherapy. In addition, the side effects of systemic chemotherapy, including neurotoxicity, myelotoxicity and gastrointestinal toxicity, may have a major impact on the quality of life of the patients and may lead to life-threatening complications (3). Therefore, identifying effective strategies that predict response to chemotherapy are required. Using these strategies, patients that are predicted to not respond to chemotherapy may receive other potentially effective treatments as early as possible and avoid unnecessary side effects. Gene expression profiling is used to predict the clinical outcome of patients with CRC (10-12). Previous studies have revealed that gene expression profiling may be used to predict cancer response to chemotherapy, including breast cancer and CRC (13-15).

The aim of the present study was to develop a predictor classifier for response to fluorouracil-based chemotherapy in patients with advanced CRC using microarray gene expression profiles of primary CRC tissues. 


\section{Materials and methods}

Data processing. The raw microarray data (CEL files) of three datasets [GSE52735 (16), GSE62080 (15) and GSE69657 (17)] and corresponding clinical data were downloaded from the Gene Expression Omnibus database (www.ncbi.nlm.nih. gov/geo). The microarray data of the 3 datasets were based on the GPL570 Affymetrix Human Genome U133 Plus 2.0 Array platform (Affymetrix; Thermo Fisher Scientific Inc., Waltham, MA, USA). The GSE52735 set contained 37 advanced CRC samples treated with a fluoropyrimidine-based chemotherapy regimen (specific chemotherapy regimens were not available). A total of 23 of the samples were classified as responders and 14 samples were classified as non-responders to the chemotherapy regimen according to Response Evaluation Criteria in Solid Tumors (RECIST) (18). The GSE62080 dataset contained 21 advanced CRC samples treated with the FOLFIRI regimen. A total of 9 samples were classified as responders and 12 samples were classified as non-responders according to the World Health Organization (WHO) criteria (19). The GSE69657 dataset contained 30 advanced CRC samples treated with the FOLFOX4 regimen. However, the raw microarray data was available for only 16 samples. A total of 7 of these samples were classified as responders and 9 samples were classified as non-responders according to RECIST. Two different evaluation criteria used in these three studies due to long time intervals between the studies, Previous studies have revealed that the RECIST criteria are comparable with the WHO criteria in evaluating the response of solid tumors (20-23). Preprocessing and normalization of the raw data were analyzed using the 'affy' (version 3.8) package (24) in R (www.r-project.org; version 3.5), using robust multi-array average for background correction and quantiles for normalization. Kernel and nearest neighbor averaging methods were used to impute the missing values using the 'impute' package (bioconductor.org/packages/impute; version 3.8) in R. The ComBat function in the 'sva' (version 3.8) package (25) was applied to remove batch effects. If one gene matched multiple probes, the average value of the probes was calculated as the expression of the corresponding gene. To build a robust predictive classifier, the GSE52735 and GSE62080 datasets were used as the training set $(n=58)$, while the GSE69657 dataset was used as the validation set $(n=16)$.

Screening of differentially expressed genes (DEGs) and enrichment analysis. Following preprocessing of the raw expression data, the DEGs between responders and non-responders in the training set were screened using the unpaired t-test in the 'limma' (version 3.8) package (26) in R. A DEG was defined as $\log _{2}$ fold change $(F C) \mid \geq 0.263$ and $P<0.05$. The Gene Ontology (GO; http://geneontology.org/) and Kyoto Encyclopedia of Genes and Genomes (KEGG; https://www. genome.jp/kegg/) pathway enrichment analyses of DEGs were performed using the 'clusterProfiler' (version 3.8) package (27) in $\mathrm{R}$ with a cut-off of $\mathrm{q}<0.01$.

Principal component analysis (PCA) prior to and following feature selection using the least absolute shrinkage and selection operator (LASSO) method. The expression values of DEGs in each sample were extracted. The LASSO logistic regression model analysis was performed using the 'glmnet' package (CRAN.R-project.org/package=glmnet; version 2.0-16) in R. The LASSO method is used to select optimal features in high-dimensional microarray data with a powerful predictive value and a low correlation between each other to prevent over-fitting (28). In the training set, the LASSO logistic regression model was used to select the optimal predictive markers. PCA using the expression profiles of the DEGs was performed prior to feature selection using the LASSO method. PCA was subsequently performed using the expression profiles of the optimal DEGs identified using by the LASSO method. Samples were plotted in two-dimensional plots across the first two principal components.

Feature selection using Boruta and random forest classifier construction. A lower-dimensional model may reduce costs and is more likely to be used by clinicians (29). Following DEGs selection by the LASSO method, a feature selection was performed using the 'Boruta' package (www. jstatsoft.org/article/view/v036i11; version 6.0.0) in R. Boruta is a random forest-based feature selection method, which provides an unbiased and stable selection of important and non-important attributes from an information system. A variable importance (VIMP) measure may be calculated and visualized based on Boruta. In the current study, DEGs selected by Boruta were used to develop a gene-based classifier for response to fluorouracil-based chemotherapy in advanced CRCs. The random forest classifier was developed using the 'randomForest' package (CRAN.R-project. org/package=randomForest; version 4.6-14) in R. The validation set (GSE69657) was used to confirm the robustness and transferability of the classifier. The performance of the classifier was assessed by accuracy, sensitivity (Se), specificity (Sp), positive predictive value (PPV), negative predictive value (NPV) and receiver operating characteristic (ROC) curves in the training and validation sets. The ROC curves were drawn and compared using the 'pROC' (version 1.13.0) package (30) in R.

\section{Results}

DEGs in responders and non-responders and enrichment analysis. The training set included 32 responders and 26 non-responders. According to the cut-off criteria $\left(\log _{2} \mathrm{FCl} \geq 0.263\right.$ and $\left.\mathrm{P}<0.05\right), 791$ genes were identified as differentially expressed between responders and non-responders. A total of 303 genes were upregulated and 488 genes were downregulated in responders. Functional enrichment analysis revealed that the biological process of DEGs were primarily involved in 'cell mitosis', 'DNA replication' and 'cell cycle' signaling pathways. The results of enrichment analysis are presented in Fig. 1.

PCA and feature selection using LASSO. For the first feature selection, LASSO logistic regression was performed using the expression data of DEGs in the training set. The group-wise classifications in 10-fold cross-validations were computed as default. A total of 31 DEGs were identified as optimal genes (Fig. 2A) with non-zero regression coefficients (Table I). 


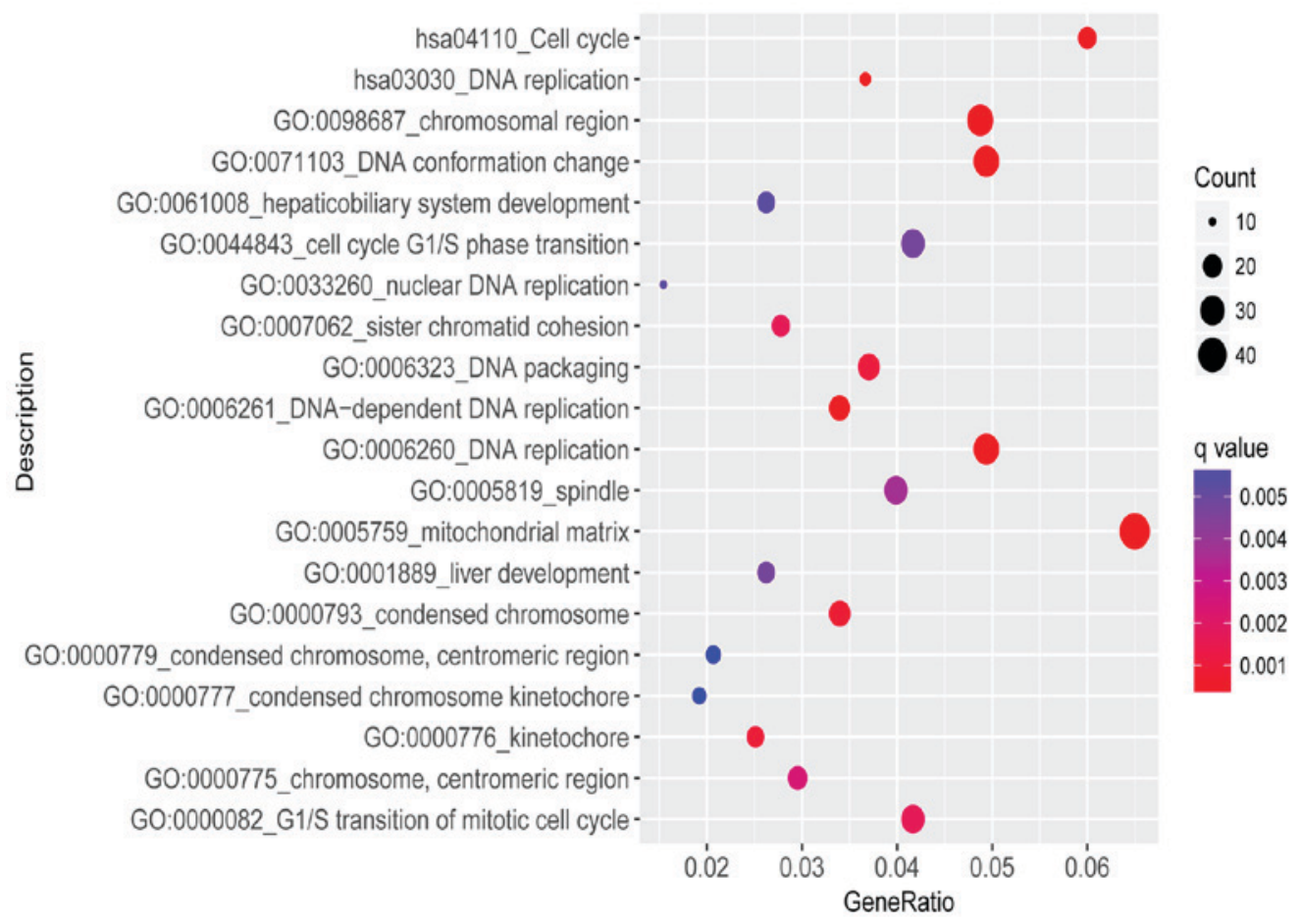

Figure 1. Significantly enriched GO annotation and enriched KEGG pathways of differentially expressed genes. GO, Gene Ontology; KEGG, Kyoto Encyclopedia of Genes and Genomes.
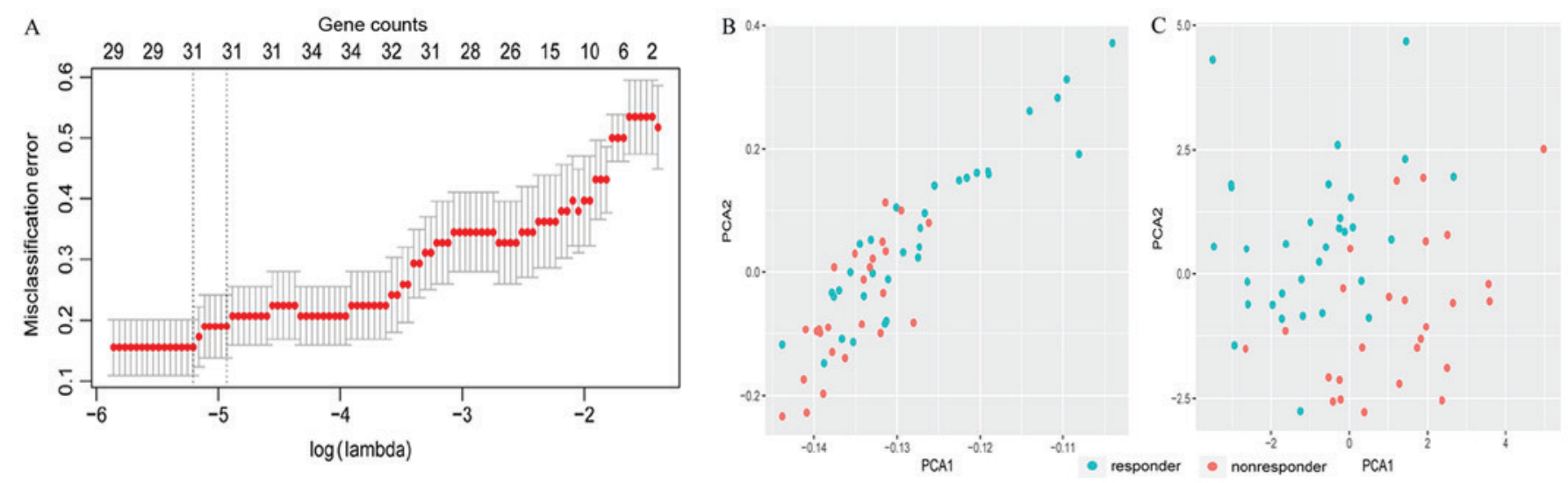

Figure 2. LASSO model and principal component analysis. (A) 10-fold cross-validation for tuning parameter selection in the LASSO model. (B) PCA prior to and (C) following LASSO variable reduction. LASSO, least absolute shrinkage and selection operator; PCA, principal component analysis.

Fig. 2B presents the results of PCA prior to feature selection using LASSO and Fig. $2 \mathrm{C}$ presents the results of PCA following feature selection using LASSO. As demonstrated in Fig. 2C, responders and non-responders are easily distinguished using the 31 DEGs selected by LASSO.

Features selection using Boruta and construct of the random forest classifier. The Boruta function was used to further select features among the 31 DEGs. A total of 13 genes were confirmed as important, 7 genes were rejected and 11 tentative genes remained (Table I). Fig. 3 presents the variables' importance. These 13 important DEGs included small muscle protein X-linked, pleckstrin homology like domain family A member 1 (PHLDA1), prostaglandin reductase 2 (PTGR2), chitinase 1 (CHIT1), histone cluster 1 H2B family member c, formin homology 2 domain containing 3, OTUD6B antisense RNA 1 (head to head), cholinergic receptor nicotinic $\beta 1$ subunit (CHRNB1), RPA interacting protein, DNA ligase 4 (LIG4), ASAP1 intronic transcript 2, small integral membrane protein 30 and c-Maf inducing protein. A random forest classifier was constructed using these 13 important DEGs.

Performance of the gene-based classifier. The accuracy of the 13-gene classifier was 0.930 in the training set and 0.810 in the validation set. Based on accuracy, Se, Sp, PPV, NPV and area under curve (AUC) values, the sample recognition efficiency of the classifier was high (Table II). ROC curve analysis revealed that the AUC was 1.000 in the training set and 0.873 in the validation set ( $\mathrm{P}=0.227$; Fig. 4). 


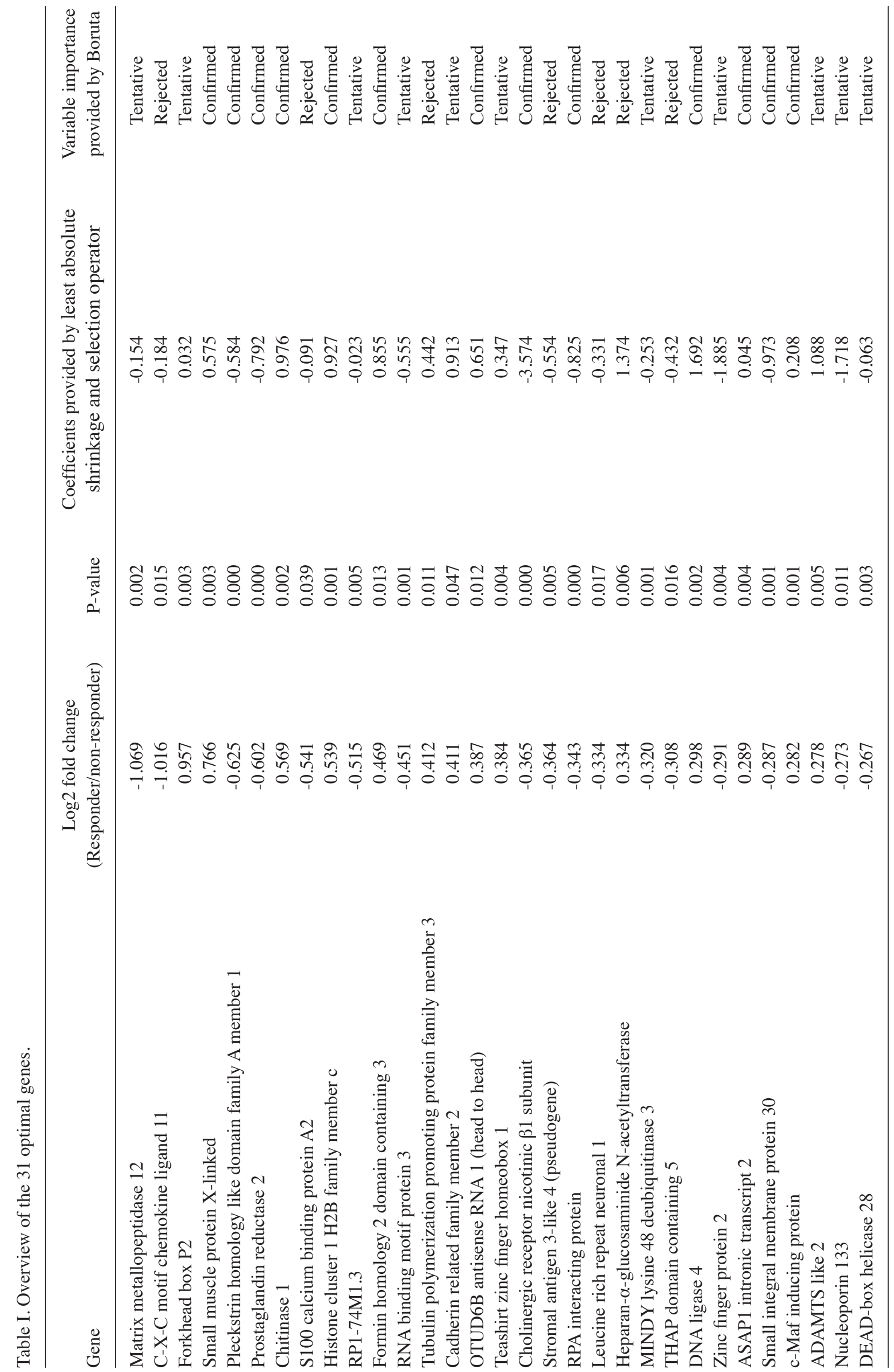


Table II. Performance of the 13-gene classifier.

\begin{tabular}{lcccccc}
\hline Cohort & Sensitivity & Specificity & $\begin{array}{c}\text { Positive } \\
\text { predictive value }\end{array}$ & $\begin{array}{c}\text { Negative } \\
\text { predictive value }\end{array}$ & Accuracy & $\begin{array}{c}\text { Area under } \\
\text { the curve }\end{array}$ \\
\hline Training set & 0.970 & 0.960 & 0.910 & 0.960 & 0.930 & 1.000 \\
Validation set & 0.860 & 0.880 & 0.750 & 0.880 & 0.810 & 0.873 \\
\hline
\end{tabular}

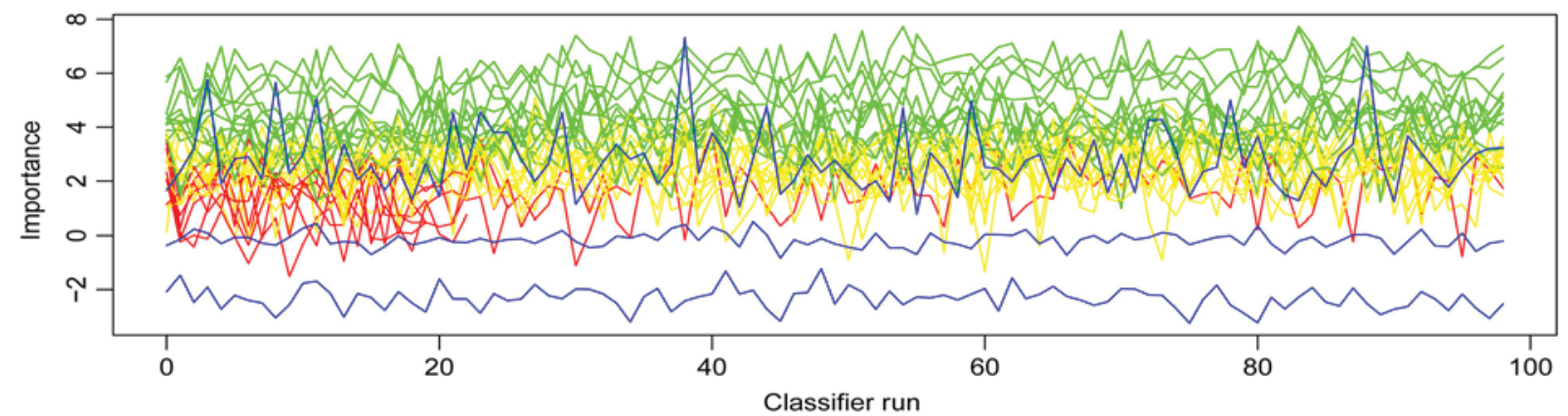

Figure 3. Z score evolution during Boruta run. Green lines correspond to confirmed attributes, yellow to tentative, red to rejected ones; and blue lines correspond to respectively minimal, average and maximal shadow attribute importance.

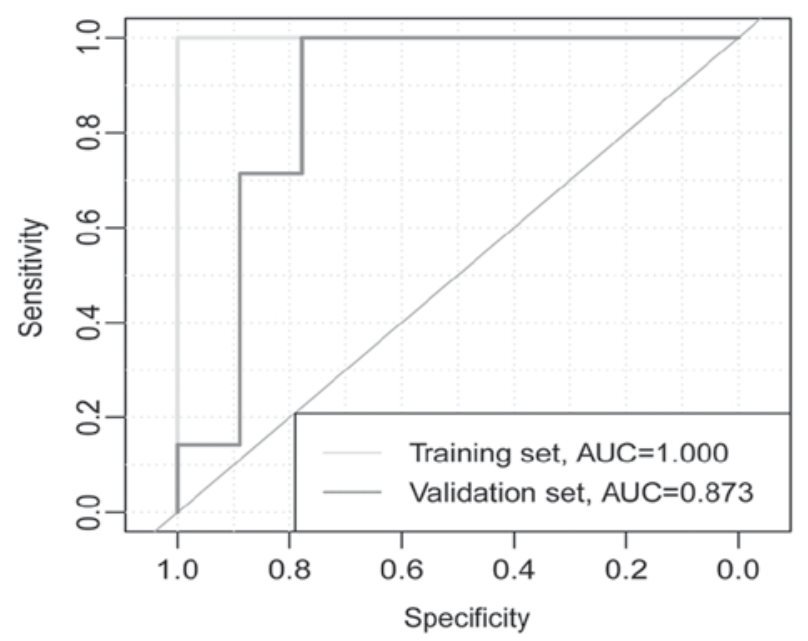

Figure 4. Receiver operating characteristic curves for training and validation sets. AUC, area under the curve.

\section{Discussion}

Personalized treatment may improve the treatment outcome of patients with tumors (31). In CRC, the gene expression levels of vascular endothelial growth factor (VEGF) and epidermal growth factor receptor (EGFR) provide the basis for selecting EGFR and VEGF inhibitor combinations (32-36). Monoclonal antibodies against VEGF and EGFR have been approved for treatment of metastatic CRC in combination with 5-FU-based regimens (3). The identification of subsets of patients that respond to specific chemotherapy regimens remains a challenge (3). A previous study demonstrated that tumors with microsatellite instability (MSI) respond well to 5-FU-based therapies; however, further studies are required to substantiate these results (37). Another previously published study suggested that MSI status does not affect the outcome of the treatment (38). Therefore, effective tools for predicting the outcome of chemotherapy are currently lacking. The present study identified 13 genes from 791 DEGs using two feature selection algorithms and developed a 13-gene predictor classifier for response to fluorouracil-based chemotherapy in CRC. The predictor classifier demonstrated high accuracy in the training and validation sets. The training set included two datasets from different centers, and the validation set was from an additional independent center. ROC curve analysis revealed that the AUC was 1.000 in the training set and 0.873 in the validation set, and their difference was not significant $(P=0.227)$. These results suggested that the classifier was robust. The study established a foundation for further research into personalized treatment of CRC.

Previous studies have attempted to identify a single biomarker to predict response to fluorouracil-based chemotherapy in CRC (17,39-42). However, there is currently no single biomarker that is routinely applied in clinical practice. CRC is a heterogeneous disease, which is compounded by changes in the molecular profile of the tumor as it progresses (3). An in vitro study demonstrated that the measurement of multiple, rather than single marker genes, may provide a more accurate assessment of drug response in colon carcinoma (43). Previous studies have been designed to identify a pattern of gene expression capable of predicting response to fluorouracil-based chemotherapy in CRC $(15,16)$. One study identified a set of 14 genes for predicting response to the FOLFIRI regimen based on 21 samples (15), and an expression profile of 7 genes was identified in another study (16). Compared with the two aforementioned studies, the current study performed a comprehensive analysis of more samples $(n=58)$ from two centers and validated the predictor classifier in an independent dataset $(n=16)$. Furthermore, to the best of the authors' knowledge, the current study is the first to construct a random forest classifier to predict response to chemotherapy in CRC. 
Considering the limited ability of Cox regression analysis to process high-dimensional data (44), it was not performed in the current study. A random forest algorithm was used to construct the classifier, which was subsequently validated with an independent dataset. The results obtained in the current study suggest that the robust classifier developed warrants further investigation.

Functional enrichment analysis revealed that certain DEGs identified in the present study are involved in DNA replication and cell cycle pathways; however, none of the 13 genes were involved in these two signaling pathways. A previous study suggested that PHLDA1 may be associated with CRC progression (45). A previous study demonstrated that PTGR2-knockdown gastric cancer cells rendered them more sensitive to cisplatin and 5-FU compared with the PTGR2-overexpressing cells (46). In addition, two variants of CHIT1, rs61745299 and rs35920428, may increase expression of the gene and have been associated with CRC (47). CHRNB1 may be a biomarker for the detection of relapsed and early relapsed CRC (48). In addition, LIG4 may mediate Wnt signaling-induced radioresistance in CRC (49). With the exception of the aforementioned studies, the association between the 13 genes identified in the current study and $\mathrm{CRC}$ or chemotherapy has not been investigated. Therefore, it is not clear whether these genes are causal or merely markers for response to fluorouracil-based chemotherapy in CRC.

Although the current study provides novel insights into the treatment of CRC, it has some limitations. The present study was based on a relatively small sample size; however, it is worth noting that the sample size in our study is relatively large compared with previous studies $(15,16)$. Future studies are required to verify and improve the 13-gene signature in a larger independent cohort of patients.

In conclusion, the current study identified a 13-gene predictor classifier for the response to fluorouracil-based chemotherapy in patients with advanced CRC.

\section{Acknowledgements}

Not applicable.

\section{Funding}

This research was supported by the Youth Science Foundation of Guangxi Medical University (grant no. GXMUYSF 201716) and the Basic Ability Enhancement Program for Young and Middle-aged Teachers of Guangxi (grant no. 2017KY0120).

\section{Availability of data and materials}

All data generated or analyzed during the present study are included in this published article.

\section{Authors' contributions}

YFL designed the study and revised the manuscript. ZHG and QYZ analyzed the data and wrote the manuscript. YL, ZHX, $\mathrm{ZH}$, and ZCC assisted with analyzing the data and writing the manuscript. All authors read and approved the final manuscript.

\section{Ethics approval and consent to participate}

Not applicable.

\section{Patient consent for publication}

Not applicable.

\section{Competing interests}

The authors declare that they have no competing interests.

\section{References}

1. Torre LA, Bray F, Siegel RL, Ferlay J, Lortet-Tieulent J and Jemal A: Global cancer statistics, 2012. CA Cancer J Clin 65: 87-108, 2015

2. Van Cutsem E, Cervantes A, Nordlinger B and Arnold D; ESMO Guidelines Working Group: Metastatic colorectal cancer: ESMO clinical practice guidelines for diagnosis, treatment and follow-up. Ann Oncol 25 (Suppl 3): iii1-iii9, 2014.

3. Brenner H, Kloor M and Pox CP: Colorectal cancer. Lancet 383: 1490-1502, 2014.

4. de Gramont A, Bosset JF, Milan C, Rougier P, Bouché O, Etienne PL, Morvan F, Louvet C, Guillot T, François E and Bedenne L: Randomized trial comparing monthly low-dose leucovorin and fluorouracil bolus with bimonthly high-dose leucovorin and fluorouracil bolus plus continuous infusion for advanced colorectal cancer: A French intergroup study. J Clin Oncol 15: 808-815, 1997.

5. Goldberg RM, Sargent DJ, Morton RF, Fuchs CS, Ramanathan RK, Williamson SK, Findlay BP, Pitot HC and Alberts SR: A randomized controlled trial of fluorouracil plus leucovorin, irinotecan, and oxaliplatin combinations in patients with previously untreated metastatic colorectal cancer. J Clin Oncol 22: 23-30, 2004.

6. Tournigand C, André T, Achille E, Lledo G, Flesh M, Mery-Mignard D, Quinaux E, Couteau C, Buyse M, Ganem G, et al: FOLFIRI followed by FOLFOX6 or the reverse sequence in advanced colorectal cancer: A randomized GERCOR study. J Clin Oncol 22: 229-237, 2004.

7. Yamazaki K, Nagase M, Tamagawa H, Ueda S, Tamura T, MurataK,Eguchi Nakajima T,Baba E, Tsuda M, Moriwaki T, et al: Randomized phase III study of bevacizumab plus FOLFIRI and bevacizumab plus mFOLFOX6 as first-line treatment for patients with metastatic colorectal cancer (WJOG4407G). Ann Oncol 27: 1539-1546, 2016

8. PetrelliF,BorgonovoK,Cabiddu M,GhilardiM,LonatiV,MasperoF, Sauta MG, Beretta GD and Barni S: FOLFIRI-bevacizumab as first-line chemotherapy in 3500 patients with advanced colorectal cancer: A pooled analysis of 29 published trials. Clin Colorectal Cancer 12: 145-151, 2013.

9. Saltz LB, Clarke S, Díaz-Rubio E, Scheithauer W, Figer A, Wong R, Koski S, Lichinitser M, Yang TS, Rivera F, et al: Bevacizumab in combination with oxaliplatin-based chemotherapy as first-line therapy in metastatic colorectal cancer: A randomized phase III study. J Clin Oncol 26: 2013-2019, 2008.

10. Wang Y, Jatkoe T, Zhang Y, Mutch MG, Talantov D, Jiang J, McLeod HL and Atkins D: Gene expression profiles and molecular markers to predict recurrence of Dukes' B colon cancer. J Clin Oncol 22: 1564-1571, 2004.

11. Eschrich S, Yang I, Bloom G, Kwong KY, Boulware D, Cantor A, Coppola D, Kruhøffer M, Aaltonen L, Orntoft TF, et al: Molecular staging for survival prediction of colorectal cancer patients. J Clin Oncol 23: 3526-3535, 2005.

12. Notterman DA, Alon U, Sierk AJ and Levine AJ: Transcriptional gene expression profiles of colorectal adenoma, adenocarcinoma, and normal tissue examined by oligonucleotide arrays. Cancer Res 61: 3124-3130, 2001.

13. Wen WH, Bernstein L, Lescallett J, Beazer-Barclay Y, Sullivan-Halley J, White M and Press MF: Comparison of TP53 mutations identified by oligonucleotide microarray and conventional DNA sequence analysis. Cancer Res 60: 2716-2722, 2000. 
14. Iwao-Koizumi K, Matoba R, Ueno N, Kim SJ, Ando A Miyoshi Y, Maeda E, Noguchi S and Kato K: Prediction of docetaxel response in human breast cancer by gene expression profiling. J Clin Oncol 23: 422-431, 2005.

15. Del Rio M, Molina F, Bascoul-Mollevi C, Copois V, Bibeau F, Chalbos P, Bareil C, Kramar A, Salvetat N, Fraslon $\mathrm{C}$, et al: Gene expression signature in advanced colorectal cancer patients select drugs and response for the use of leucovorin, fluorouracil, and irinotecan. J Clin Oncol 25 773-780, 2007.

16. Estevez-Garcia P, Rivera F, Molina-Pinelo S, Benavent M, Gómez J, Limón ML, Pastor MD, Martinez-Perez J, Paz-Ares L, Carnero A and Garcia-Carbonero R: Gene expression profile predictive of response to chemotherapy in metastatic colorectal cancer. Oncotarget 6: 6151-6159, 2015.

17. Li S, Lu X, Chi P and Pan J: Identification of HOXB8 and KLK11 expression levels as potential biomarkers to predict the effects of FOLFOX4 chemotherapy. Future Oncol 9: 727-736, 2013.

18. Therasse P, Arbuck SG, Eisenhauer EA, Wanders J, Kaplan RS, Rubinstein L, Verweij J, Van Glabbeke M, van Oosterom AT, Christian MC and Gwyther SG: New guidelines to evaluate the response to treatment in solid tumors. European Organization for Research and Treatment of Cancer, National Cancer Institute of the United States, National Cancer Institute of Canada. J Natl Cancer Inst 92: 205-216, 2000.

19. Miller AB, Hoogstraten B, Staquet $M$ and Winkler A: Reporting results of cancer treatment. Cancer 47: 207-214, 1981.

20. Aras M, Erdil TY, Dane F, Gungor S, Ones T, Dede F, Inanir S and Turoglu HT: Comparison of WHO, RECIST 1.1, EORTC and PERCIST criteria in the evaluation of treatment response in malignant solid tumors. Nucl Med Commun 37: 9-15, 2016.

21. Khokher S, Qureshi MU and Chaudhry NA: Comparison of WHO and RECIST criteria for evaluation of clinical response to chemotherapy in patients with advanced breast cancer. Asian Pac J Cancer Prev 13: 3213-3218, 2012.

22. Choi JH, Ahn MJ, Rhim HC, Kim JW, Lee GH, Lee YY and Kim IS: Comparison of WHO and RECIST criteria for response in metastatic colorectal carcinoma. Cancer Res Treat 37: 290-293, 2005

23. Park JO, Lee SI, Song SY, Kim K, Kim WS, Jung CW, Park YS, Im YH, Kang WK, Lee MH, et al: Measuring response in solid tumors: Comparison of RECIST and WHO response criteria. Jpn J Clin Oncol 33: 533-537, 2003.

24. Gautier L, Cope L, Bolstad BM and Irizarry RA: affy-analysis of Affymetrix GeneChip data at the probe level. Bioinformatics 20 : 307-315, 2004

25. Leek JT, Johnson WE, Parker HS, Jaffe AE and Storey JD: The sva package for removing batch effects and other unwanted variation in high-throughput experiments. Bioinformatics 28 : 882-883, 2012

26. Ritchie ME, Phipson B, Wu D, Hu Y, Law CW, Shi W and Smyth GK: limma powers differential expression analyses for RNA-sequencing and microarray studies. Nucleic Acids Res 43: e47, 2015

27. Yu G, Wang LG, Han Y and He QY: clusterProfiler: An R package for comparing biological themes among gene clusters. OMICS 16: 284-287, 2012

28. Wu TT, Chen YF, Hastie T, Sobel E and Lange K: Genome-wide association analysis by lasso penalized logistic regression. Bioinformatics 25: 714-721, 2009.

29. Cuyle PJ and Prenen H: Current and future biomarkers in the treatment of colorectal cancer. Acta Clin Belg 72: 103-115, 2017.

30. Robin X, Turck N, Hainard A, Tiberti N, Lisacek F, Sanchez JC and Müller M: pROC: An open-source package for R and S+ to analyze and compare ROC curves. BMC Bioinformatics 12: 77, 2011.

31. Stintzing S: Personalized treatment for colorectal carcinomas Dtsch Med Wochenschr 142: 1652-1659, 2017 (In German).

32. Gustavsson B, Carlsson G, Machover D, Petrelli N, Roth A, Schmoll HJ, Tveit KM and Gibson F: A review of the evolution of systemic chemotherapy in the management of colorectal cancer. Clin Colorectal Cancer 14: 1-10, 2015

33. Iwamoto $S$, Takahashi $T$, Tamagawa $H$, Nakamura $M$, Munemoto Y, Kato T, Hata T, Denda T, Morita Y, Inukai M, et al: FOLFIRI plus bevacizumab as second-line therapy in patients with metastatic colorectal cancer after first-line bevacizumab plus oxaliplatin-based therapy: The randomized phase III EAGLE study. Ann Oncol 26: 1427-1433, 2015.
34. Bazarbashi S, Aljubran A, Alzahrani A, Mohieldin A, Soudy $\mathrm{H}$ and Shoukri M: Phase I/II trial of capecitabine, oxaliplatin, and irinotecan in combination with bevacizumab in first line treatment of metastatic colorectal cancer. Cancer Med 4: 1505-1513, 2015.

35. Heinemann V, von Weikersthal LF, Decker T, Kiani A, Vehling-Kaiser U, Al-Batran SE, Heintges T, Lerchenmüller C, Kahl C, Seipelt G, et al: FOLFIRI plus cetuximab versus FOLFIRI plus bevacizumab as first-line treatment for patients with metastatic colorectal cancer (FIRE-3): A randomised, open-label, phase 3 trial. Lancet Oncol 15: 1065-1075, 2014.

36. Stremitzer S, Sebio A, Stintzing S and Lenz HJ: Panitumumab safety for treating colorectal cancer. Expert Opin Drug Saf 13: 843-851, 2014

37. Saridaki Z, Souglakos J and Georgoulias V: Prognostic and predictive significance of MSI in stages II/III colon cancer. World J Gastroenterol 20: 6809-6814, 2014.

38. Webber EM, Kauffman TL, O'Connor E and Goddard KA: Systematic review of the predictive effect of MSI status in colorectal cancer patients undergoing 5FU-based chemotherapy. BMC Cancer 15: 156, 2015.

39. Nakanishi R, Kitao H, Fujinaka Y, Yamashita N, Iimori M, Tokunaga E, Yamashita N, Morita M, Kakeji Y and Maehara Y: FANCJ expression predicts the response to 5-fluorouracil-based chemotherapy in MLH1-proficient colorectal cancer. Ann Surg Oncol 19: 3627-3635, 2012.

40. Simmer F, Venderbosch S, Dijkstra JR, Vink-Börger EM, Faber C, Mekenkamp LJ, Koopman M, De Haan AF, Punt CJ and Nagtegaal ID: MicroRNA-143 is a putative predictive factor for the response to fluoropyrimidine-based chemotherapy in patients with metastatic colorectal cancer. Oncotarget 6: 22996-23007, 2015.

41. Molina-Pinelo S, Carnero A, Rivera F, Estevez-Garcia P, Bozada JM, Limon ML, Benavent M, Gomez J, Pastor MD, Chaves M, et al: MiR-107 and miR-99a-3p predict chemotherapy response in patients with advanced colorectal cancer. BMC Cancer 14: 656, 2014.

42. Candy PA, Phillips MR, Redfern AD, Colley SM, Davidson JA, Stuart LM, Wood BA, Zeps N and Leedman PJ: Notch-induced transcription factors are predictive of survival and 5-fluorouracil response in colorectal cancer patients. Br J Cancer 109: 1023-1030, 2013.

43. Mariadason JM, Arango D, Shi Q, Wilson AJ, Corner GA, Nicholas C, Aranes MJ, Lesser M, Schwartz EL and Augenlicht LH: Gene expression profiling-based prediction of response of colon carcinoma cells to 5-fluorouracil and camptothecin. Cancer Res 63: 8791-8812, 2003.

44. Kursa MB and Rudnicki WR: Feature selection with the boruta package. J Stat Soft 36: 1-13, 2010.

45. Zhang $Z$ and Huang J: Intestinal stem cells-types and markers Cell Biol Int 37: 406-414, 2013.

46. Chang EY, Tsai SH, Shun CT, Hee SW, Chang YC, Tsai YC, Tsai JS, Chen HJ, Chou JW, Lin SY and Chuang LM: Prostaglandin reductase 2 modulates ROS-mediated cell death and tumor transformation of gastric cancer cells and is associated with higher mortality in gastric cancer patients. Am J Pathol 181: 1316-1326, 2012.

47. Li FF, Yan P, Zhao ZX, Liu Z, Song DW, Zhao XW, Wang XS, Wang GY and Liu SL: Polymorphisms in the CHIT1 gene: Associations with colorectal cancer. Oncotarget 7: 39572-39581, 2016.

48. Chang YT, Yeh YS, Ma CJ, Huang CW, Tsai HL, Huang MY, Cheng TL and Wang JY: Optimization of a multigene biochip for detection of relapsed and early relapsed colorectal cancer. J Surg Res 220: 427-437, 2017.

49. Jun S, Jung YS, Suh HN, Wang W, Kim MJ, Oh YS, Lien EM, Shen X, Matsumoto Y, McCrea PD, et al: LIG4 mediates Wnt signalling-induced radioresistance. Nat Commun 7: 10994, 2016.

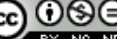

This work is licensed under a Creative Commons Attribution-NonCommercial-NoDerivatives 4.0 International (CC BY-NC-ND 4.0) License. 